\title{
"It's Like Riding Out the Chaos": Caring for Socially Complex Patients in an Ambulatory Intensive Care Unit (A-ICU)
}

Brian Chan, MD, MPH ${ }^{1,2}$

Elizabeth Hulen, $M A^{3,4}$

Samuel Edwards, $M D, M P H^{1,3}$

Matthew Mitchell, MTS 2

Christina Nicolaidis, $M D, M P H^{1,5,6}$

Somnath Saba, MD, MPH $H^{1,3}$

'Division of General Internal Medicine and Geriatrics, Oregon Health \& Science University, Portland, Oregon

${ }^{2}$ Central City Concern, Portland, Oregon

${ }^{3}$ Center to Improve Veteran Involvement in Care, VA Portland Health Care System, Portland, Oregon

${ }^{4}$ Department of Sociology, Portland State University, Portland, Oregon

${ }^{5}$ School of Social Work, Portland State University, Portland, Oregon

${ }^{6}$ School of Public Health, Oregon Health \& Science University and Portland State University, Portland, Oregon

Conflicts of interest: authors report none.

\section{CORRESPONDING AUTHOR}

Brian Chan, MD, MPH

Assistant Professor, Division of General Internal Medicine and Geriatrics,

Oregon Health and Science University 3181 SW Sam Jackson Park Road L475

Portland, OR 97239-3098

chanbri@ohsu.edu

\begin{abstract}
PURPOSE High-need high-cost (HNHC) patients consume a large proportion of health resources but often receive suboptimal care in traditional primary care. Intensive ambulatory care interventions attempt to better meet these patients' needs, but we know little about how teams delivering these interventions in clinics serving socially complex patient populations perceive their work.
\end{abstract}

METHODS We performed a qualitative study of multidisciplinary staff experiences at a Federally Qualified Health Center (FQHC) caring for predominantly homeless HNHC patients in the context of an ongoing implementation of an ambulatory intensive care unit (A-ICU) intervention. We conducted semistructured interviews with 9 ambulatory intensive care team members and 6 "usual care" members. We conducted a thematic analysis, using an inductive approach, at a semantic level.

RESULTS Staff viewed complexity as a combination of social, behavioral, and medical challenges that lead to patient-health care system mismatch. Staff perceive the following as key ingredients in caring for HNHC patients: addressing both psychosocial and clinical needs together; persistence in staying connected to patients through chaotic periods; shared commitment and cohesion among interdisciplinary team members; and flexibility to tailor care to patients' individual situations. Participants' definitions of success focused more on improving patient engagement than reducing utilization or cost.

CONCLUSION FQHC staff working with HNHC patients perceive mismatch between the health care system and patients' clinical and social needs as the key driver of poor outcomes for these patients. Intensive ambulatory care teams may bridge mismatch through provision of psychosocial supports, flexible care delivery, and fostering team cohesion to support patient engagement.

Ann Fam Med 2019;17:495-501. https://doi.org/10.1370/afm.2464.

\section{PURPOSE}

$\mathrm{H}$ igh-need high-cost $(\mathrm{HNHC})$ patients use a large proportion of health care resources, ${ }^{1}$ but receive poor-quality care. ${ }^{2}$ Persons experiencing poverty make up a large share of HNHC patients. ${ }^{3,4}$ These patients struggle with health needs, citing among others financial, physical, and emotional impacts of illness, and inadequate social support. ${ }^{5}$ Providers also struggle to care for these patients, citing patients' psychosocial barriers, untreated mental illness, and substance use disorders (SUDs), as well as system issues of lack of time, poor care coordination with specialists, and fragmented care. ${ }^{6-8}$ These factors overwhelm primary care capacity. ${ }^{7,9,10}$

A variety of interventions attempt to address HNHC patients. ${ }^{11,12}$ One approach changes primary care by creating expanded, multidisciplinary teams designed to address the needs of HNHC patients-an example is the "ambulatory intensive care unit" (A-ICU). Common features of A-ICUs include (1) decreased panel sizes of selected "high-risk" 
patients, (2) additional personnel for care coordination activities, and (3) appreciation of and integration with mental health and case management professionals to address psychosocial needs and streamline care. ${ }^{13,14}$ While evidence is mixed on the efficacy of these models ${ }_{1}^{15}$ patients in A-ICUs perceive benefit from navigation of financial and social needs by these teams. ${ }^{16}$ Studies to date, however, have not explored the inner workings of A-ICUs or the perspectives of multidisciplinary providers working in these care models. Understanding the perspectives of clinical staff caring for socially complex patients offers insights for improving A-ICU design. Therefore, we conducted a qualitative analysis of multidisciplinary staff caring for HNHC patients to identify what team members perceive as (1) the principal needs and barriers they are addressing, and (2) the key ingredients for addressing patients' needs.

\section{METHODS}

\section{Setting and Brief Intervention Description}

Old Town Clinic (OTC) is a federally qualified health center (FQHC) serving over 5,000 low-income patients with high rates of homelessness and SUDs in Portland, Oregon. In addition to primary care services, OTC provides co-located mental health services delivered by psychiatric nurse practitioners; SUD counseling services; chronic disease pharmacists and onsite pharmacy and laboratory services; wellness activities; and social work services and community health workers (CHWs). Despite this multidisciplinary care, a subset of OTC patients continues to consume a large share of clinician and staff attention and have high rates of emergency department (ED) visits and hospitalizations. This spurred the development of the Streamlined Unified Meaningfully Managed Interdisciplinary Team ("SUMMIT") A-ICU.

SUMMIT is a multi-component primary care A-ICU featuring low patient-to-staff ratios, flexible scheduling, and outreach activities. ${ }^{17}$ Starting in fall of 2015, OTC providers were able to refer patients to SUMMIT if they met utilization criteria: 1 or more hospitalizations in prior 6 months, with multiple medical or mental health conditions or poor engagement in care as outlined in Table 1. The SUMMIT team then reviewed individual cases to determine those most in need of intensive intervention. Team activities included initial comprehensive intake with medical and behavioral health team members, patient-driven health goal setting, post-hospital transitional care protocols, medication management assessment, weekly panel review, and co-visits for mental health counseling and case management to address unmet needs. The team has flexibility to conduct outreach activities, facilitate unscheduled clinic visits, accompany patients to specialty appointments, and deliver addiction treatment. Approximately 100 patients were enrolled in SUMMIT by spring 2017, when we began this study.

\section{Study Design and Interview Participants}

We invited all OTC staff (clinicians, medical assistants, care team managers, health assistants, pharmacists, licensed clinical social workers [LCSW] and CHWs) to participate. We interviewed all members of the SUMMIT team, including 2 physicians, 2 care coordinators, 2 LCSWs, a pharmacist, a nurse, and a care team manager. We conducted 6 additional interviews with members of the same clinic that were part of "usual care," including 1 primary care clinician, 2 CHWs, an LCSW with mental health counseling roles, a chronic disease pharmacist, and a care team manager. A total of 15 OTC clinic staff participated.

\section{Data Collection}

One research team member (E.H.) conducted interviews with each participant between April and August of 2017, with each interview lasting an average of 45 minutes. Interviews were conducted using a semistructured guide (Supplemental Appendix, available at http://www.AnnFamMed.org/content/17/6/495/suppl/ DC1/) that addressed perspectives on defining complex patients, staff experiences delivering care for $\mathrm{HCHN}$ patients, the impact of SUMMIT, and questions on team structure, psychological safety, and staff experience. All interviews were audio recorded, transcribed,

\section{Table 1. Enrollment Criteria for Referral to the SUMMIT Ambulatory ICU}

Enrollment criteria

1 or more medical/surgical hospitalizations in last 6 months And either

2 or more of the following medical conditions

Chronic kidney disease state III or higher

Congestive heart failure

Chronic obstructive pulmonary disease, Gold group C/D

Diabetes with $\mathrm{A}_{1 \mathrm{c}}>8 \%$

End stage liver disease

Osteomyelitis/severe soft-tissue infection

1 of the above medical condition, plus 1 or more of the following comorbid conditions

Mental health condition

Substance use disorder

1 of the above medical conditions, plus difficulty engaging in usual primary care (missed appointments)

Gold = Global Initiative for Chronic Obstructive Lung Disease; $C / D=$ Gold group C or D; ICU = intensive care unit; SUMMIT = streamlined unified meaningfully managed interdisciplinary team. 
and transferred to ATLAS.ti version 8 (Scientific Software Development $\mathrm{GmbH}$ ) for analysis.

\section{Data Analysis}

We conducted a thematic analysis using an inductive approach at a semantic level. Our approach included familiarization with the transcripts; generation of initial codes, identifying themes and subthemes; development of a conceptual thematic map; and writing the report. ${ }^{18}$ First, 3 authors (B.C., S.E., E.H.) reviewed several transcripts and generated memos for potential codes. This group met 3 times to discuss memos and develop a preliminary codebook through an iterative, consensusbased process. Next, 2 members of the team (B.C. and E.H.) applied the codebook independently to 2 transcripts and met to compare codes, resolve differences, and refine the codebook. We then coded all transcripts using 3 dyads consisting of 4 authors (B.C., E.H., S.E., M.M.); 1 author (E.H.) participated in all the dyads for consistency. Next, 2 members of the research team (B.C. and E.H.) generated thematic maps, then met with senior members of the research team (S.S., C.N., S.E.) to refine themes and identify exemplars. We combined data from participants in different professional roles, including members of SUMMIT and "usual care" teams, because our goal was to discover general themes in caring for complex patients that incorporated a range of perspectives. Conceptual theme maps and exemplars were then shown to the entire research team for review and critique before finalizing. The Oregon Health \& Science University Institutional Review Board approved the study procedures.

\section{RESULTS}

We focused on themes that could serve as useful lessons in how staff perceive care for HNHC patients and how A-ICUs function. The themes fell into 3 domains, 2 that mapped to our research questions about defining complexity and how care teams can support complex patients, and a third domain relating to how these team members viewed success in working with HNHC patients (Figure 1).

\section{Domain 1: What Makes Patients Complex}

Theme 1: Multiple Dimensions of Complexity Participants noted non-medical factors that contributed to patient complexity in this population, including poverty, mental illness, and SUDs that, in addition to their medical issues, created high treatment burdens requiring more time to address than is traditionally allotted in primary care.

"We talk about social complexity...comorbid substance use disorders, homelessness, low health literacy, and poverty really can complicate the real-world execution of treatment plan[s] ... for people who have medical complexity" (SUMMIT Pbysician)

\section{Theme 2: Patient-System} Mismatch

FQHC staff caring for complex patients also perceived patientsystem mismatch as a contributor to complexity. Mismatch arises from patients' social circumstances limiting access to services, behavioral issues interfering with care engagement, and lack of health system flexibility to address these barriers. A staff member reflected on the inability for a patient to receive services due to homelessness:

"He was homeless when we made the referral and doesn't... fit into [the] standard hospice system.... Health care systems 
are designed for these neat packages of people that are housed, have family support, have access to other resources, are not actively using substances.... The services aren't really designed for complex folks, so that can be really frustrating and exhausting." (SUMMIT LCSW)

"Our health care system is one-size-fits-all glove... Everyone is supposed to get the same care and I feel frustration of having to try to take care of patients that I just couldn't do it in 20 minutes. They had so many more needs than I could meet." (Usual Care Pbysician)

\section{Domain 2: Key Ingredients Staff Perceive as Important in Caring for HNHC Patients}

Staff believed that effectively assisting these patients involves addressing both clinical and psychosocial needs together, building connection with patients through acceptance of the chaos that often affects their lives, shared commitment among team members, and flexibility in care delivery.

\section{Theme 3: Addressing Both Psychosocial and Clinical Needs}

Participants noted that it wasn't possible to separate provision of psychosocial support from traditional medical care. This can run counter to what occurs in usual care.

"I spent an hour with a patient last week and we didn't talk about medical problems.... It was a therapeutic session. I'm not a trained therapist, but [that's] what it was. We didn't talk about diabetes. We didn't talk about her foot ulcers....A lot of times we end up doing the work of social workers, but when you do primary care, you have to do that. It's not 'oh hold on,... I'm not getting into that. I'm only here for the medical stuff.' It all wraps up into one." (SUMMIT Physician)

\section{Theme 4: Staying Connected to Patients Through Chaotic Periods}

Staff perceived the opportunity to have repeated engagement and the time to persist in building relationships as necessary for these patients. Persistence was seen as essential in gaining trust.

"Having more time, and really having this ability to ride through chaos with people where we are chasing people down and showing up at their apartment multiple times until we finally catch them when they're home...drawing people into the fold of care and riding out chaos with them." (SUMMIT LCSW)

Team members endorsed the importance of sticking with patients amidst chaotic situations as a way to develop trusting relationships.

"I think once we gain trust, people start to do a little better because they realize we're going to stick with them." (SUMMIT Nurse)
Sticking with patients and being there for them through challenging circumstances was an intervention itself.

"...We are dealing with impossible situations and the best we can do is just sit with our patients through that and be there." (SUMMIT Physician)

"I think sometimes there is a lot of angst around 'are we doing enough for this person' without recognizing that being there and facing something with somebody is quite a bit of an intervention." (SUMMIT Physician)

\section{Theme 5: Team Cohesion and Shared Commitment to the Patient}

Team members caring for HNHC patients noted the importance of shared values and commitment, citing mutual respect for other disciplines and appreciation of the need for teamwork.

"We respect one another's clinical view... We come at this from different backgrounds and feel like we get more out of our patient care experience if we hear what everyone else has to say.... We have a very supportive and inclusive team environment" (SUMMIT Physician)

The importance of the team comes through particularly when patients aren't faring as well as hoped:

"They [other team members] really listen and they really care and we all really feel it when someone does fail...or something bad happens. It's a very empathetic group of people...." (SUMMIT Nurse)

In addition, the ability for a team to be small and nimble was seen as a strength as it allowed for increased cohesion.

"One of the issues with complex care is [it's] spread out amongst a bunch of different people.... There's a learning curve each time the patient meets with a different provider... With SUMMIT, it's a small team.... Everybody knows what's going on with the patients in terms of their conditions and it really cuts through the confusion." (Usual Care LCSW)

\section{Theme 6: Flexibility in Care Delivery}

The ability to tailor care to patients' individual needs was another ingredient staff members felt they provided to complex patients. A SUMMIT care coordinator described a strategy to assist patients with attending specialty appointments:

"I'll have appointments with patients just with myself if patients need help with scheduling outside the clinic and scheduling transportation.... If a patient chronically no-shows to a (specialty) appointment... I'1l make an appointment for them to come [see] me and we'll schedule together and... give them an appointment planner or write up all their appointments for them." 
Another example of how teams tailor care to the individual patient was utilizing different team members' skillsets based on the patient's needs at a given time.

"It's a huge umbrella-it's social work, it's case management, it's a little bit of counseling, a little bit of behavioral health. It's a mixture of all those things depending on what the person needs." (Usual Care $\mathrm{CHW})$

\section{Domain 3: Defining Success in Caring for HNHC Patients}

A third domain encompassing a final theme arose from the interviews about how teams caring for HNHC patients define success. How team members gauged success emerged in the interviews describing what staff enjoyed most about working with HNHC patients, and perceptions of how multidisciplinary care teams improve quality of care.

\section{Theme 7: Supporting Patient Engagement With Care Teams}

Improved interactions and engagement with the clinic were important markers of success for care team members. Staff defined success through observing changes in patient self-care behaviors, patient interactions with the clinic, and in the staff-patient relationship.

"By us being able to adapt how we are giving him care, he's become engaged with the clinic in a different way that is better than it was before. [Before] he wasn't getting his needs met. Now he comes in frequently... his life is much less chaotic." (SUMMIT Pbysician)

"...The patients that have been on SUMMIT for a while who have a really solid relationship with us, that makes a huge difference. They are able to call. They are telling us what their needs are. They can make it to appointments and...coordinate all of those needs a little bit better when they know that we're going to be reliable and [here] is where they can come for help." (SUMMIT Pbysician)

The development of trust and connection between patient and care team was also described as a marker of success.

"...To see the level of support that a lot of their clients are getting and the breakthroughs they've been able to have and that trust and relationship built when it was so difficult before...." (Usual Care LCSW)

Though the SUMMIT intervention was developed as a way to address high ED and hospital utilization, staff members did not mention reduced utilization as a marker of success.

"We are dealing with a pretty sick population.... These are patients that maybe do need to be in the hospital... A hospitalization is not necessarily a bad outcome for a lot of these patients." (SUMMIT Physician)

\section{DISCUSSION}

In this study of multidisciplinary staff perspectives caring for HNHC patients experiencing homelessness, we set out to learn how teams perceive patient complexity and the activities they undertake to care for socially complex patients. We found that care teams perceived social and behavioral factors as compounding medical issues that create individual complexity; this, coupled with health care system inflexibility, creates a patientsystem mismatch that reinforces barriers to care for this population. Staff felt that addressing both psychosocial and clinical needs of patients, persisting with patients through life chaos, having team cohesion and shared commitment to the patients, and flexibility in care delivery are important to caring for HNHC patients. Finally, staff perceived success as improved patient engagement.

A prior study of primary care clinicians' perspectives on caring for complex patients found that health system factors (eg, payment systems, care fragmentation) and local system factors (eg, insufficient clinical support, communication with specialists, productivity demands) impeded optimal care delivery. ${ }^{9}$ Our study extends these findings by assessing care of complex patients in the context of an A-ICU intervention. A-ICUs address system complexity by attending to local system environments, by providing additional time and staff devoted to consolidation or coordination of care, and appreciation of reduced panel sizes and flexible scheduling.

Our findings align with existing theories of patient complexity that recognize the contributions of both individual patient factors and the health system environment in creating mismatch. ${ }^{19,20}$ Teams caring for complex patients appreciate this mismatch and attempt to address it by: providing psychosocial support in concert with addressing clinical need, by maintaining connection with patients during chaotic periods, by being flexible in care delivery and patient engagement, and by doing this in a team-based manner that appreciates the multiple dimensions of complexity. These findings complement existing program descriptions by giving insight into what activities staff find helpful in support of their HCHN patients. We found common characteristics seen in these models: adaptable teams that provide enhanced psychosocial supports for patients (through use of embedded nursing, social workers, etc as care team members), low patient-to-staff ratios with flexible schedules, and high-touch patient-centered approaches to care. ${ }^{13-15}$ In addition, our findings suggest teams caring for HNHC patients may need to persist through the social chaos of patients' lives that often results in non-engagement with primary care, missed appointments, or failed transition plans, to build trusting relationships and achieve succcess. ${ }^{9}$ 
Our finding that staff members value a sense of unity and esprit de corps speaks to the value of cohesive multidisciplinary teams doing this work. As prior studies have shown, individual members of multidisciplinary teams may have different conceptualizations of which disciplines are part of a care team-often these are only a team in name. ${ }^{21}$ Our findings speak to the importance of co-located, embedded teams that "hear what everyone else has to say."

Our final finding suggests that A-ICU team members view their proximal goal as increased engagement rather than reducing utilization. As a recent study highlighted ${ }^{22}$ success of intensive outpatient programs may be predicated on the ability to improve patient participation in their health care. Their findings of how intensive primary care programs improve engagement parallel our findings: the importance of multidisciplinary team composition, having requisite time and resources, and flexible approaches to patient goals. For staff caring for socially and medically complex patients, it may be necessary to experience increased hospitalization in order to manage previously unaddressed medical needs, while developing the trust and engagement necessary for long-term reductions in utilization. Comorbid SUD, mental illness, and/or poverty, along with patients' medical conditions may make meeting well-intentioned clinical practice guidelines impractical or unachievable. Development of more relevant measures may be needed for these patients; Kaiser Permanente Colorado describes how incorporating stakeholders (patients, clinicians, and payers) improved care guidelines for their complex patients. ${ }^{23}$ Likewise, HIV care models found increased engagement to be associated with improved clinical outcomes. ${ }^{24}$

This study has several limitations. We interviewed clinical staff from a single-site FQHC that is already well resourced and serves HNHC patients with high rates of homelessness and SUD. As such, our study offers unique insights into the care of some of the most socially complex patients in our health care system. As with most qualitative research, we were not aiming to achieve broad generalizability but hope that our findings are transferable to other sites caring for HNHC patients with social complexity. Participants in the interviews were likely self-selecting and may differ from other staff members who care for such patients.

Our findings have some important implications. First, flexible approaches for addressing patientsystem mismatch appear necessary to address the needs of HNHC patients. This argues against protocolized care in favor of general principles that permit team adaptability to meet the needs of patients and their environments. Future intervention design may need to account for the high chaos and unpredict- able engagement patterns of complex patients. The next step is to test whether these elements lead to increased patient engagement, and self-efficacy, and improved quality of care. Even if these programs do not reduce overall cost or utilization, they may still provide high value if they are cost neutral but enhance the quality of care delivered. ${ }^{25}$

Health care teams caring for medically and socially complex patients endorse the importance of addressing patient-system mismatches, providing psychosocial supports in midst of patient's life chaos, and celebrating increased patient engagement with primary care. These principles should guide the evolution and design of future interventions to care for HNHC patients.

To read or post commentaries in response to this article, see it online at http://www.AnnFamMed.org/content/17/6/495.

Key words: patient-centered care; primary care redesign; primary care; vulnerable populations; underserved populations

Submitted November 29, 2018; submitted, revised, May 13, 2019; accepted May 24, 2019.

Funding support: Dr Chan was supported by grant number K12HS022981 from the Agency for Healthcare Research and Quality (AHRQ). Dr Edwards was supported by VA Health Services Research $\&$ Development grant CDA 16-152.

Previous presentations: Results from this study were presented as an oral abstract at the national Society for General Internal Medicine meeting; April 11-14, 2018; Denver, Colorado.

Acknowledgments: Patricia Carney, PhD, for early guidance on codebook development and Rachel Solotaroff, MD, MCR, for partnered support of SUMMIT. We would like to thank the clinical staff who participated in this research and who continue to support implementation of SUMMIT.

Supplemental materials: Available at http://www.AnnFamMed. org/content/17/6/495/suppl/DC1/.

\section{References}

1. Cohen S, Yu W. The Concentration and Persistence in the Level of Health Expenditures over Time: Estimates for the U.S. Population, 2008-2009. Statistical Brief \#354. Rockville, MD: Agency for Healthcare Research and Quality; 2012.

2. Vogeli $C$, Shields AE, Lee TA, et al. Multiple chronic conditions: prevalence, health consequences, and implications for quality, care management, and costs. J Gen Intern Med. 2007;22(Suppl 3): 391-395.

3. Bell J, Turbow S, George M, Ali MK. Factors associated with highutilization in a safety net setting. BMC Health Serv Res. 2017;17(1): 273.

4. Kushel MB, Vittinghoff E, Haas JS. Factors associated with the health care utilization of homeless persons. JAMA. 2001;285(2): 200-206.

5. Koch G, Wakefield BJ, Wakefield DS. Barriers and facilitators to managing multiple chronic conditions: a systematic literature review. West J Nurs Res. 2015;37(4):498-516. 
6. Loeb DF, Binswanger IA, Candrian C, Bayliss EA. Primary care physician insights into a typology of the complex patient in primary care. Ann Fam Med. 2015;13(5):451-455.

7. Okunogbe A, Meredith LS, Chang ET, Simon A, Stockdale SE, Rubenstein LV. Care coordination and provider stress in primary care management of high-risk patients. J Gen Intern Med. 2018; 33(1):65-71.

8. Lin EH, Katon W, Von Korff M, et al. Frustrating patients: physician and patient perspectives among distressed high users of medical services. J Gen Intern Med. 1991;6(3):241-246.

9. Loeb DF, Bayliss EA, Candrian C, deGruy FV, Binswanger IA. Primary care providers' experiences caring for complex patients in primary care: a qualitative study. BMC Fam Pract. 2016;17:34.

10. Kim LY, Rose DE, Soban LM, et al. Primary care tasks associated with provider burnout: findings from a Veterans Health Administration survey. J Gen Intern Med. 2018;33(1):50-56.

11. Peikes D, Chen A, Schore J, Brown R. Effects of care coordination on hospitalization, quality of care, and health care expenditures among Medicare beneficiaries: 15 randomized trials. JAMA. 2009; 301(6):603-618.

12. Smith SM, Wallace E, O'Dowd T, Fortin M. Interventions for improving outcomes in patients with multimorbidity in primary care and community settings. Cochrane Database Syst Rev. 2016;3: CD006560.

13. Bodenheimer T. Center for Health Care Strategies. Strategies to reduce costs and improve care for high-utilizing Medicaid patients: reflections on pioneering programs. https://www.chcs.org/resource/ strategies-to-reduce-costs-and-improve-care-for-high-utilizingmedicaid-patients-reflections-on-pioneering-programs/. Published Oct 2013. Accessed Aug 1, 2016.

14. Hong CS, Siegel AL, Ferris TG. Caring for high-need, high-cost patients: what makes for a successful care management program? Issue Brief (Commonw Fund). 2014;19:1-19.

15. Edwards ST, Peterson K, Chan B, Anderson J, Helfand M. Effectiveness of intensive primary care interventions: a systematic review. J Gen Intern Med. 2017;32(12):1377-1386.
16. Ganguli I, Orav EJ, Weil E, Ferris TG, Vogeli C. What do high-risk patients value? perspectives on a care management program. J Gen Intern Med. 2018;33(1):26-33.

17. Chan B, Edwards ST, Devoe M, et al. The SUMMIT ambulatory-ICU primary care model for medically and socially complex patients in an urban federally qualified health center: study design and rationale. Addict Sci Clin Pract. 2018;13(1):27.

18. Braun V, Clarke V. Using thematic analysis in psychology. Qual Res Psychol. 2006;3(2):77-101.

19. Shippee ND, Shah ND, May CR, Mair FS, Montori VM. Cumulative complexity: a functional, patient-centered model of patient complexity can improve research and practice. J Clin Epidemiol. 2012; 65(10):1041-1051

20. Safford MM, Allison JJ, Kiefe Cl. Patient complexity: more than comorbidity. the vector model of complexity. J Gen Intern Med. 2007:22(Suppl 3):382-390.

21. Doekhie KD, Buljac-Samardzic M, Strating MMH, Paauwe J. Who is on the primary care team? Professionals' perceptions of the conceptualization of teams and the underlying factors: a mixed-methods study. BMC Fam Pract. 2017;18(1):111.

22. Zulman DM, O'Brien CW, Slightam C, Breland JY, Krauth D, Nevedal AL. Engaging high-need patients in intensive outpatient programs: a qualitative synthesis of engagement strategies. J Gen Intern Med. 2018;33(11):1937-1944.

23. Bennett WL, Robbins CW, Bayliss EA, et al. Engaging stakeholders to inform clinical practice guidelines that address multiple chronic conditions. J Gen Intern Med. 2017;32(8):883-890.

24. Mugavero MJ, Davila JA, Nevin CR, Giordano TP. From access to engagement: measuring retention in outpatient HIV clinical care. AIDS Patient Care STDS. 2010;24(10):607-613.

25. Yoon J, Chang E, Rubenstein LV, et al. Impact of primary care intensive management on high-risk veterans' costs and utilization: a randomized quality improvement trial. Ann Intern Med. 2018;168(12): 846-854. 